\title{
On Electrogravity Duality*
}

\author{
Naresh Dadhich ${ }^{\dagger}$ \\ Inter-University Centre for Astronomy \& Astrophysics, \\ Post Bag 4, Ganeshkhind, Pune - 411 007, India.
}

\begin{abstract}
By resolving the gravitational field into electric and magnetic parts, we define an electrogravity duality transformation and discover an interesting property of the field. Under the duality transformation a vacuum/flat spacetime maps into the original spacetime with a topological defect of global monopole/texture. The elctrogravity-duality is thus a topological defect generating process. It turns out that all black hole solutions possess dual solutions that imbibe a global monopole.
\end{abstract}

\footnotetext{
${ }^{*}$ Received Honorable Mention in the 1998 Gravity Research Foundation Essay Competition

${ }^{\dagger}$ E-mail : nkd@iucaa.ernet.in
} 
It is well-known that gravitational field can, in analogy with the electromagnetic field, be resolved into electric and magnetic parts [1-5]. Electric part is caused by charge (source) while magnetic part is due to motion of charge. In the case of gravity it would be mass-energy that would serve as charge that would produce electric part and its motion would give rise to magnetic part. Unlike other fields, gravitation has two kinds of charges, the one the usual matter-energy and the other is the gravitational field energy. They will lead to further decomposition of electric part into active (the former, the usual Coulombic) and passive (the latter producing space curvature). For electromagnetic decomposition of gravitation, we resolve the Riemann curvature into electric and magnetic parts reletive to a timelike unit vector. Electric and magnetic parts are described by second rank 3-tensors. Each of active and passive electric parts is described by a symmetric tensor while magnetic part consists of a symmetric Weyl magnetic part and an antisymmetric part representing energy flux.

By elctrogravity duality we would mean interchange of active and passive electric parts of the field. It turns out that it is equivalent to interchange of the Ricci and the Einstein curvatures. The vacuum equation would however remain invariant under the duality transformation. The most interesting vacuum solutions are the black hole solutions and they are also unique. In obtaining balck hole solutions, it turns out that one does not need to use all the equations; i.e. there remains one equation free which is implied by the others. For example in the simplest case of the Schwarzschild solution the vacuum equation ultimately reduces to the two equations, $\nabla^{2} \phi=0$ and $(r \phi)^{\prime}=0 g_{00}=-g_{11}^{-1}=1+2 \phi(r)$, and a prime denotes derivative w.r.t. $r$. Clearly the latter equation implies the former. That is even if we modify the vacuum equation by putting something on the r.h.s. of the former, the equations will still yield the Schwarzschild as the unique solution. Since the Schwarzschild solution is unique, the modified equation could as well characterize vacuum for spherical symmetry. The modified equation would now no longer be duality-invariant. The interesting question that arises is, what does the solution of the dual set (dual-vacuum) represent? It turns out that the dual solution represents the Schwarzschild black hole with a global monopole charge [6]. Similarly it is possible to find solutions dual to the Kerr and NUT solutions [7-8]. The duality transformation would thus generate global monopole charge for vacuum (including electrovac) solutions in spherical and axial symmetries. 
As for vacuum, flat spacetime could as well be characterized by a dulaity non-invariant eqaution. In the static case spacetime dual to flat spacetime reperesents a massless pure monopole (zero mass limit of the solution dual to the Schwarzschild black hole) while for the homogeneous isotropic non-static case it is given by an FRW model with the equation of state $\rho+3 p=0$, which characterizes a global texture.

Global monopoles and textures are stable topological defects. They are supposed to be produced when global symmetry is spontaneously broken in phase transitions in the early Universe [9]. It is remarkable that the duality transformation automatically incorporates these topological defects in the solutions of the Eistein equation in a natural way. The elctrogravity-duality thus generates topological defects in the vacuum/flat solutions of the Einstein equation. This is a remarkable new property.

The main aim of this essay is to expose how the duality transformation automatically includes the global monopole for the stationary vacuum solutions and global texture for flat spacetime. We shall demonstrate this for the Schwarzschild solution and flat spacetime. The same procedure would however work for the Kerr-NUT solutions.

Let us begin with the resolution of the Riemann curvature relative to a unit timelike vector, as follows :

$$
\begin{gathered}
E_{a c}=R_{a b c d} u^{b} u^{d}, \tilde{E}_{a c}=* R *_{a b c d} u^{b} u^{d} \\
H_{a c}=* R_{a b c d} u^{b} u^{d}=H_{(a c)}-H_{[a c]}
\end{gathered}
$$

where

$$
\begin{gathered}
H_{(a c)}=* C_{a b c d} u^{b} u^{d} \\
H_{[a c]}=\frac{1}{2} \eta_{a b c e} R_{d}^{e} u^{b} u^{d} .
\end{gathered}
$$


Here $C_{a b c d}$ is the Weyl conformal curvature, $\eta_{a b c d}$ is the 4-dimensional volume element. $E_{a b}=E_{b a}, \tilde{E}_{a b}=\tilde{E}_{b a},\left(E_{a b}, \tilde{E}_{a b}, H_{a b}\right) u^{b}=0, H=H_{a}^{a}=0$ and $u^{a} u_{a}=1$. The Ricci tensor could then be written as

$$
R_{a}^{b}=E_{a}^{b}+\tilde{E}_{a}^{b}+(E+\tilde{E}) u_{a} u^{b}-\tilde{E} g_{a}^{b}+\frac{1}{2}\left(\eta_{a m n} H^{m n} u^{b}+\eta^{b m n} H_{m n} u_{a}\right)
$$

where $E=E_{a}^{a}$ and $\tilde{E}=\tilde{E}_{a}^{a}$. It may be noted that $E=\left(\tilde{E}+\frac{1}{2} T\right) / 2$ defines the gravitational charge density while $\tilde{E}=-T_{a b} u^{a} u^{b}$ defines the energy density relative to the unit timelike vector $u^{a}$.

The vacuum equation, $R_{a b}=0$ is in general equivalent to

$$
\text { E or } \tilde{E}=0, H_{[a b]}=0=E_{a b}+\tilde{E}_{a b}
$$

which is symmetric in $E_{a b}$ and $\tilde{E}_{a b}$.

We define the duality transformation as

$$
E_{a b} \longleftrightarrow \tilde{E}_{a b}, H_{a b}=H_{a b} .
$$

Thus the vacuum eqaution (6) is invariant under the duality transformation (7). From eqn. (1) it is clear that the duality transformation would map the Ricci tensor into the Einstein tensor and vice-versa. This is because contraction of Riemann is Ricci while of its double dual is Einstein.

For obtaining the Schwarzschild solution, we consider the metric,

$$
d s^{2}=c^{2}(r, t) d t^{2}-a^{2}(r, t) d r^{2}-r^{2}\left(d \theta^{2}+\sin ^{2} \theta d \varphi^{2}\right) .
$$

The natural choice for the resolving vector in this case is of course it being hypersurface orthogonal, pointing along the $t$-line. From eqn. (6), $H_{[a b]}=0$ and $E_{2}^{2}+\tilde{E}_{2}^{2}=0$ lead to $a c=1$ (for this, no boundary condition of asymptotic flatness need be used). Now $\tilde{E}=0$ gives $a=(1-2 M / r)^{-1 / 2}$, which is the Schwarzschild solution. Note that we did not need to use the remaining equation $E_{1}^{1}+\tilde{E}_{1}^{1}=0$, it is hence free and is implied by the rest. Without affecting the Schwarzschild solution, we can introduce some distribution in the 1-direction. 
We hence write the alternate equation as

$$
H_{[a b]}=0=\tilde{E}, E_{a b}+\tilde{E}_{a b}=\lambda w_{a} w_{b}
$$

where $\lambda$ is a scalar, and $w_{a}$ is a spacelike unit vector parallel to the (radial) acceleration. It is clear that it will also admit the Schwarzschild solution as the general solution, and it determines $\lambda=0$. That is for spherical symmetry the above alternate equation also characterizes vacuum, because the Schwarzschild solution is unique.

Let us now employ the duality transformation (7) to the above equation (9) to write

$$
H_{[a b]}=0=E, E_{a b}+\tilde{E}_{a b}=\lambda w_{a} w_{b} .
$$

Its general solution for the metric (8) is given by

$$
c=a^{-1}=\left(1-2 k-\frac{2 M}{r}\right)^{1 / 2} .
$$

This is the Barriola-Vilenkin solution [6] for the Schwarzschild particle with global monopole charge, $\sqrt{2 k}$. Again we shall have $a c=1$ and $E=0$ will then yield $c=(1-2 k-2 M / r)^{1 / 2}$ and $\lambda=2 k / r^{2}$. This has non-zero stresses given by

$$
T_{0}^{0}=T_{1}^{1}=\frac{2 k}{r^{2}}
$$

A global monopole is described by a triplet scalar, $\psi^{a}(r)=\eta f(r) x^{a} / r, x^{a} x^{a}=$ $r^{2}$, which through the usual Lagrangian generates energy-momentum distribution at large distance from the core precisely of the form given above in (12) [6]. Like the Schwarzschild solution the monopole solution (11) is also the unique solution of eqn.(10).

If we translate eqns. (9) and (10) in terms of the familiar Ricci components, they would read as

$$
R_{0}^{0}=R_{1}^{1}=\lambda, R_{2}^{2}=0=R_{01}
$$

and 


$$
R_{0}^{0}=R_{1}^{1}=0=R_{01}, R_{2}^{2}=\lambda .
$$

For the metric (8), eqns. $R_{0}^{0}=R_{1}^{1}=R_{01}=0$ lead to $a c=1$ and $c^{2}=f(r)=$ $1+2 \phi$, say, and then

$$
\begin{gathered}
R_{0}^{0}=-\nabla^{2} \phi \\
R_{2}^{2}=-\frac{2}{r^{2}}(r \phi)^{\prime} .
\end{gathered}
$$

Now the set (13) integrates to give $\phi=-M / r$ and $\lambda=0$, which is the Schwarzschild solution while (14) will give $\phi=-k-M / r$ and $\lambda=2 k / r^{2}$, the Schwarzschild with global monopole charge. Thus global monopole owes its existence to the constant $k$, appearing in the solution of the usual Laplace equation implied by eqns. (14) and (15). It defines a pure gauge for the Newtonian theory, which could be chosen freely, while the Einstein vacuum equation determines it to be zero. For the dual-vacuum equation (14), it is free like the Newtonian case but it produces non-zero curvature and hence would represent non-trivial physical and dynamical effect (see $R_{2}^{2}=-2 k / r^{2} \neq 0$ unless $k=0$ ). This is the crucial difference between the Newtonian theory and GR in relation to this problem, that the latter determines the relativistic potential $\phi$ absolutely, vanishing only at infinity. This freedom is restored in the dual-vacuum equation, of course at the cost of introducing stresses that represent a global monopole charge. The uniform potential would hence represent a massless global monopole $(M=0$ in the solution (11)), which is solely supported by the passive part of electric field. It has been argued and demonstrated [5] that it is the non-linear aspect of the field (which incorporates interaction of gravitational field energy density) that produces space-curvatures and consequently the passive electric part. It is important to note that the relativistic potential $\phi$ plays the dual role of the Newtonian potential as well as the non-Newtonian role of producing curvature in space. The latter aspect persisits even when potential is constant different from zero. It is the dual-vacuum equation that uncovers this aspect of the field.

On the other hand, flat spacetime could also in alternative form be characterized by

$$
\tilde{E}_{a b}=0=H_{[a b]}, E_{a b}=\lambda w_{a} w_{b}
$$


leading to $c=a=1$, and implying $\lambda=0$. Its dual will be

$$
E_{a b}=0=H_{[a b]}, \tilde{E}_{a b}=\lambda w_{a} w_{b}
$$

yielding the general solution,

$$
c^{\prime}=a^{\prime}=0 \Longrightarrow c=1, a=\text { const. }=(1-2 k)^{-1 / 2}
$$

which is non-flat and represents a global monopole of zero mass, as it follows from the solution (11) when $M=0$. This is also the uniform relativistic potential solution.

Further it turns out that a perfect fluid with $\rho+3 p=0$ goes to flat spacetime under the duality transformation [3]. This is the equation of state, which means $E=0$, that characterizes global texture [7,19]. That is, the necessary condition for spacetimes of topological defects; global textures and monopoles is $E=0$. The non-static homogeneous and isotropic (here $w_{a}$ is an isotropic unit vector) solution of the dual-flat equation (18) is the FRW metric with $\rho+3 p=0$, which determines the scale factor $S(t)=\alpha t+\beta$, and $\rho=3\left(\alpha^{2}+n\right) /(\alpha t+\beta)^{2}, n= \pm 1,0$. This is also the unique solution. The general solutions of the dual-flat equation are thus the massless global monopole (uniform potential) spacetime in the static case and the global texture spacetime in the non-static homogeneous and isotropic case. Thus they are dual to flat spacetime.

The above method would straightway work for the charged black hole as well as for the de Sitter spacetime. In the latter case the duality only changes the sign of the cosmological constant, i.e. de Sitter to anti de Sitter. Though the calculations are considerably more involved for the Kerr and NUT solutions, the prescription works and the solutions dual to them have been found [7-8]. We have thus constructed electrogravity-dual solutions to all the black hole solutions including the NUT solution as well as to flat spacetime representing massless global monopole and global texture.

This procedure of constructing dual solutions would work so long as there occurs a free equation in the Einstein set which is not used in finding the solution. Note that this is so for all the black hole solutions. Then the dual set admits a solution like the original set which incorporates a topological defect 
in the original spacetime. This is a remarkable property of the gravitational field, which is exposed by the duality transformation. The duality transformation for a fluid source has been considered [10], and it turns out that a fluid solution maps into a fluid solution with $\rho \leftrightarrow(\rho+3 p) / 2, p \leftrightarrow(\rho-p) / 2$, and heat flux and pressure anisotropy remaining unaltered. This shows that the stiff fluid is dual to dust, $\rho+3 p=0$ is as expected dual to flat spacetime, and the radiation and the de Sitter models are self dual.

It could be argued that duality-related spacetimes do share the basic gravitational property at the Newtonain level. Could the duality transformation hence signify "maximal closeness" or "minimal difference" between them? Then dual-flat spacetimes would be "minimally" curved. By modifying the vacuum/flat equation we have been able to construct solutions dual to black holes and flat spacetime. The duality transformation only produces a topological defect in terms of global monopole/texture in the original spacetime. This appears to be an interesting property of the field. What good it is in terms of its applications remains to be investigated. At any rate it is a novel way of creating topological defects in the vacuum/flat spacetimes.

Acknowledgement: I thank L.K. Patel for fruitful collaboration for finding the dual-Kerr solution and Mohammad Nouri-Zonoz and Donald LyndenBell for the dual-NUT solution. 


\section{References}

[1] C Lanczos 1938 Ann.Math. 39842.

[2] L Bell 1959 C R Acad. Sci. 2481297.

[3] M A G Bonilla and J M M Senovilla 1997 GRG 2991.

[4] N Dadhich 1997 gr-qc/9712021.

[5] 1998 to be submitted.

[6] M Barriola and A Vilenkin 1989 Phys.Rev.Lett. 63341.

[7] N Dadhich and L K Patel 1998 to be submitted.

[8] M Nouri-Zonoz, N Dadhich and D Lynden-Bell 1998 to be submitted.

[9] A Vilenkin and E P S Shellard 1994 Cosmic Strings and Other Topological Defects (Cambridge University Press).

[10] N Dadhich and L K Patel 1998 gr-qc/9801076 Class Quantum Grav Lett. 15 L27. 